\title{
Conectando la Teoría con la Práctica en Asignaturas de Ingeniería Química
}

\author{
Santiago Rodríguez-Pérez y Carmen Arnáiz \\ Universidad de Sevilla, Escuela Politécnica Superior, Departamento de Ingeniería Química y Ambiental. \\ Virgen de África 7, 41011 Sevilla-España (e-mail: santiagorp@us.es, mcarnaiz@us.es)
}

Recibido Jun. 11, 2013; Aceptado Jul. 24, 2013; Versión final recibida Sep. 10, 2013

\begin{abstract}
Resumen
Este trabajo presenta el diseño de una asignatura virtual de Operaciones Básicas en Ingeniería Química de pregrado y su implementación en la plataforma WebCT. La opinión, uso e impacto en el rendimiento académico de un grupo de alumnos es analizado mediante un cuestionario de satisfacción. Esta información permitió determinar la percepción positiva de los estudiantes hacia los contenidos visuales como complemento importante en el estudio de las materias. Sin embargo, no usaron activamente la herramienta visual en su aprendizaje, pero esto no se tradujo negativamente en el rendimiento de los estudiantes. Demasiadas clases presenciales, demasiadas actividades y tareas, así como un nivel homogéneo medio-alto de los estudiantes son las causas que explicarían la poca incidencia en este curso del apoyo virtual.
\end{abstract}

Palabras clave: enseñanza-aprendizaje, TICs, ingeniería química, plataforma educativa, rendimiento académico

\section{Connecting Theory with the Practice in Courses of Chemical Engineering}

\begin{abstract}
This paper presents the design of a virtual undergraduate course of Unit Operations in Chemical Engineering and its implementation in the WebCT platform. The opinion, use and impact on the academic performance of a group of students are analyzed through a questionnaire. This information allowed determining the positive perception of the students about the visual contents as an important complement of learning. However, the students did not actively use the visual tool while studying the course subjects, but this did not significantly impact the academic performance of the students. It seems that too many face-toface classes, too much homework, and the medium-high level of the group of students would explain these results.
\end{abstract}

Keywords: teaching-learning, ICT, chemical engineering, virtual learning environment, academic performance, 


\section{INTRODUCCIÓN}

La Universidad de Sevilla (US) ha adaptado todos sus estudios al Espacio Europeo de Educación Superior (EEES). Con el reconocimiento de Campus de Excelencia Internacional, la oferta académica incluye 79 Titulaciones de Grado y Dobles Grados, 86 Másteres Oficiales y más de 150 Títulos Propios (US, 2013). Entre las Titulaciones mejor valoradas se encuentran los Grados de Ingeniería en la Escuela Politécnica Superior (EPS). Los Grados en Ingeniería Eléctrica, Electrónica Industrial, Mecánica y Química Industrial sustituyen a los estudios de Ingeniería Técnica Industrial en sus diferentes especialidades. Las Titulaciones de Ingeniería Técnica Industrial son de las más demandadas en el mercado de trabajo ya que se encuentran, respecto del total de la oferta de empleo universitario, entre las cinco más solicitadas de forma continuada (datos correspondientes a los cursos académicos 07-08, 08-09, 09-10, 10-11), superando la oferta de empleo al número de egresados que terminan su formación (EPS, 2013).

Los estudios de Grado en Ingeniería Química Industrial capacitan al graduado para trabajar como Ingeniero Técnico Industrial con especial énfasis en Ingeniería Química: calcular, diseñar, construir y controlar plantas químicas y desarrollar sistemas de gestión ambiental y de calidad. Este Grado se está impartiendo desde el año académico 2010-2011. Actualmente, están implementados el primer, segundo y tercer curso. Sin embargo, algunas modificaciones son necesarias para poder alcanzar excelentes resultados académicos pues, en determinadas materias, se ha separado la teoría de la práctica y ambas se imparten en diferentes cuatrimestres. Por esta razón, muchos estudiantes tienen problemas para aprender y conectar los conocimientos teórico-prácticos de estas disciplinas. Este es el caso de la asignatura Operaciones Básicas (OB) y Experimentación en Ingeniería Química I (EIQ1), cuyos descriptores académicos incluyen balances de materia y energía, flujo de fluidos, transferencia de calor y transferencia de masa. En la asignatura OB se imparten estos contenidos desde un punto de vista teórico (incluyendo problemas) y en EIQ1 desde un punto de vista experimental (prácticas de laboratorio). No obstante, $\mathrm{OB}$ se imparte en el primer cuatrimestre del tercer año y EIQ1 en el segundo cuatrimestre del mismo año. Por lo tanto, las prácticas, que tanto ayudan al alumno a comprender la teoría, son posteriores a la asignatura teórica, dificultando el aprendizaje del alumno. Por otra parte, hay alumnos que cursan la asignatura experimental sin haber cursado la teórica, con lo que alcanzan con mayor dificultad los objetivos de aprendizaje, al carecer de los fundamentos teóricos para la realización de las prácticas y la posterior redacción del informe de las mismas.

En la actualidad, son varios los autores que investigan la aplicación de las Tecnologías de la Información y Comunicación (TIC) para mejorar el proceso de aprendizaje (García y García, 2008, Maceiras et al, 2010). La comprensión de los conceptos de Ingeniería Química es un reto debido a su naturaleza teórico-práctica (Solsona et al., 2003). Facilitar al alumnado la asimilación de los conceptos teóricos y los procesos en los que se pueden observar es un objetivo para todos los profesionales en la educación superior universitaria. En los últimos años las TIC se han integrado en múltiples asignaturas del área de la ingeniería química, mostrando ventajas e inconvenientes (Barak, 2007). Entre las principales ventajas, se encuentra el desarrollo de nuevas estrategias de enseñanza para las sesiones prácticas, mediante la utilización de ilustraciones, videos, simulaciones, enlaces con entornos externos vía internet, etc. (Domingues et al., 2010). El proyecto Europeo Library of Labs (2011) ofrece varios laboratorios virtuales para experimentos de física, matemáticas, química, ingeniería y ciencias de la computación. Los laboratorios virtuales de Ingeniería Química incluyen ejemplos de reactores no ideales, y la dinámica del proceso y de control (Komulainen et al., 2012). Babich y Mavrommatis (2009) estudian la integración de experimentos virtuales y trabajos de laboratorio real poniendo de manifiesto que las TIC no pueden cubrir toda la metodología pedagógica para la enseñanza en la ingeniería química moderna. Se llega al consenso de que una combinación de métodos tradicionales de enseñanza y las TIC es el camino a seguir. Esta integración de las TIC en la metodología tradicional de enseñanza parece permitir un estudio integral de los procesos tecnológicos de alta complejidad.

Aunque las TIC no son la panacea para solucionar cualquier problema educativo, actualmente, constituyen una herramienta esencial en los procesos de enseñanza-aprendizaje (Jung, 2005). El objetivo de este trabajo fue la elaboración de un material audiovisual de las prácticas de EIQ1 que permita reforzar las clases de teoría de $\mathrm{OB}$, conectando la teoría con la práctica para un aprendizaje más efectivo. La ventaja de la utilización de estos métodos de aprendizaje es que el alumno puede disponer de ellos siempre que lo necesite, independientemente del lugar en el que se encuentre.

\section{METODOLOGÍA}

Para la elaboración de este trabajo se identificaron las partes esenciales de las prácticas de la asignatura EIQ1, para relacionarlas con los conceptos teóricos de la asignatura OB. Se diseñaron la estructura y las secuencias de grabación, necesarias para el montaje de los vídeos de 9 prácticas de laboratorio. Se realizó la grabación de todas las secuencias en el Laboratorio de Experimentación de Ingeniería Química del 
Departamento de Ingeniería Química y Ambiental de la EPS. Las prácticas de laboratorio se grabaron mientras eran realizadas por los alumnos durante el curso académico 2010-2011. El periodo de grabación de las secuencias necesarias fue de 9 días durante las sesiones de prácticas ordinarias de la asignatura EIQ1, con el consentimiento de los alumnos, de 4 horas de duración por sesión. Tras la grabación de las secuencias de vídeo, se procedió al montaje de cada una de las prácticas, combinando imágenes y texto de manera sincronizada. El montaje de los vídeos de cada una de las prácticas se ha llevado a cabo, utilizando los programas: Windows Movie Maker, Power Point y Windows Movie Player. El producto final son 9 vídeos, que permiten al alumno de la asignatura $\mathrm{OB}$, ver la aplicación de los conceptos teóricos en el laboratorio. El material audiovisual se implementó en la asignatura de OB en la plataforma de enseñanza virtual de la US (WebCT) durante el curso académico 2012-2013, como material complementario de la asignatura.

Las Plataformas de Enseñanza Virtual suelen proporcionar herramientas para apoyar la metodología pedagógica utilizada, incluyendo los objetivos de aprendizaje, los módulos de aprendizaje (temas, problemas, bibliografía obligatoria y recomendada), las presentaciones que se muestran en las clases presenciales, la recepción de trabajos de los alumnos, la administración de grupos de estudiantes (prácticas, seminarios, trabajos en grupo), exámenes virtuales, cuestionarios de satisfacción, tutorías online, la comunicación entre estudiantes y entre estudiantes y profesores (foros) y contenidos complementarios y/o enlaces externos. Además, permite el seguimiento del proceso de aprendizaje y rendimiento de cada alumno en tareas específicas (Martín-Blas y Serrano-Fernández, 2009; Jyothi et al., 2012).

La Universidad de Sevilla adoptó desde el curso 2006/7 la Plataforma de Enseñanza Virtual (WebCT) como herramienta que permite incorporar las TIC como apoyo a la docencia. La elaboración de la estrategia corporativa de e-learning ha sido desarrollada, mediante la creación del nuevo Área de Universidad Digital, en 2006, dentro del Servicio de Informática y Comunicaciones (SIC) y la coordinación con otros servicios universitarios: Instituto de Ciencias de la Educación (ICE), Secretariado de Recursos Audiovisuales (SAV), Secretariado de Postgrado, entre otros (León et al. 2008). Sólo durante el curso 2010-2011 el servicio de Enseñanza Virtual de la US ha sido utilizado por más de 61.000 alumnos y 3.100 profesores, con una oferta que supera los 2.800 cursos (Sánchez, L. 2011).

La asignatura $\mathrm{OB}$ en WebCT está estructurada en diversas herramientas. En la página principal se presentan todos los contenidos para comodidad del alumno. A través de ella se accede a las siguientes carpetas:

1.- Programa. Contiene el programa docente oficial de la asignatura para el curso.

2.-Guía de aprendizaje. Contiene unas recomendaciones de estudio de la asignatura.

3.- Objetivos específicos. Incluye los objetivos de aprendizaje por temas.

4.- Temas. Recoge los contenidos teóricos de cada tema, la colección de problemas y la bibliografía obligatoria y recomendada.

5.- Actividades académicamente dirigidas. Contiene los trabajos propuestos de distintas partes de la asignatura a lo largo del curso que deben ser realizados por grupos de 3 alumnos.

6.- Presentaciones Power Point. Aporta recursos didácticos para apoyar las clases magistrales y guiar el estudio.

7.- Calificaciones. Carpeta de consulta de las notas de las distintas partes evaluables de la asignatura.

8.- Documentación adicional. Material específico de algunos temas. Incluye formulario.

9.- Exámenes. Esta carpeta incluye algunos exámenes de teoría y problemas. En estos últimos se indica la solución correcta. También aparece la documentación que el alumno debe llevar al examen para poder realizar los problemas (ábacos, diagramas y tablas).

10.- Material audiovisual. Contiene, por primera vez en el curso académico 12-13, vídeos de las prácticas de la asignatura EIQ 1, todas ellas íntimamente relacionadas con OB.

Al finalizar el curso se sometió a la opinión de los alumnos el interés de esta iniciativa y su uso mediante cuestionario de opinión. El cuestionario constaba de 3 preguntas de respuesta cerrada (Sí/NO): la primera para evaluar el grado de interés del material audiovisual complementario; la segunda indagaba sobre el uso por parte del alumnado de dicho material a lo largo de la asignatura; la última pregunta recogía posibles causas por las que el alumnado no haya utilizado el material audiovisual (falta de tiempo, exceso de material u otros motivos (que debía especificar en respuesta abierta).

Los participantes del estudio fueron la totalidad de los alumnos matriculados por primera vez en la asignatura, es decir, se excluyeron los alumnos que abandonaron el Plan de Estudios 2001 para continuar su formación en el Plan de Estudios 2010, pues tenían la asignatura EIQ1 aprobada. En definitiva, 17 alumnos fueron encuestados sobre un total de 23. Hay que puntualizar que se trata del primer año en que se imparte esta asignatura, dentro del nuevo plan de estudios. De ahí, el bajo número de alumnos matriculados. 


\section{RESULTADOS Y DISCUSIÓN}

Las principales características del material audiovisual elaborado son el enfoque específico, su autonomía y el grupo de destinatarios al que va dirigido. El procedimiento para acceder al contenido audiovisual se muestra en la Figura 1.

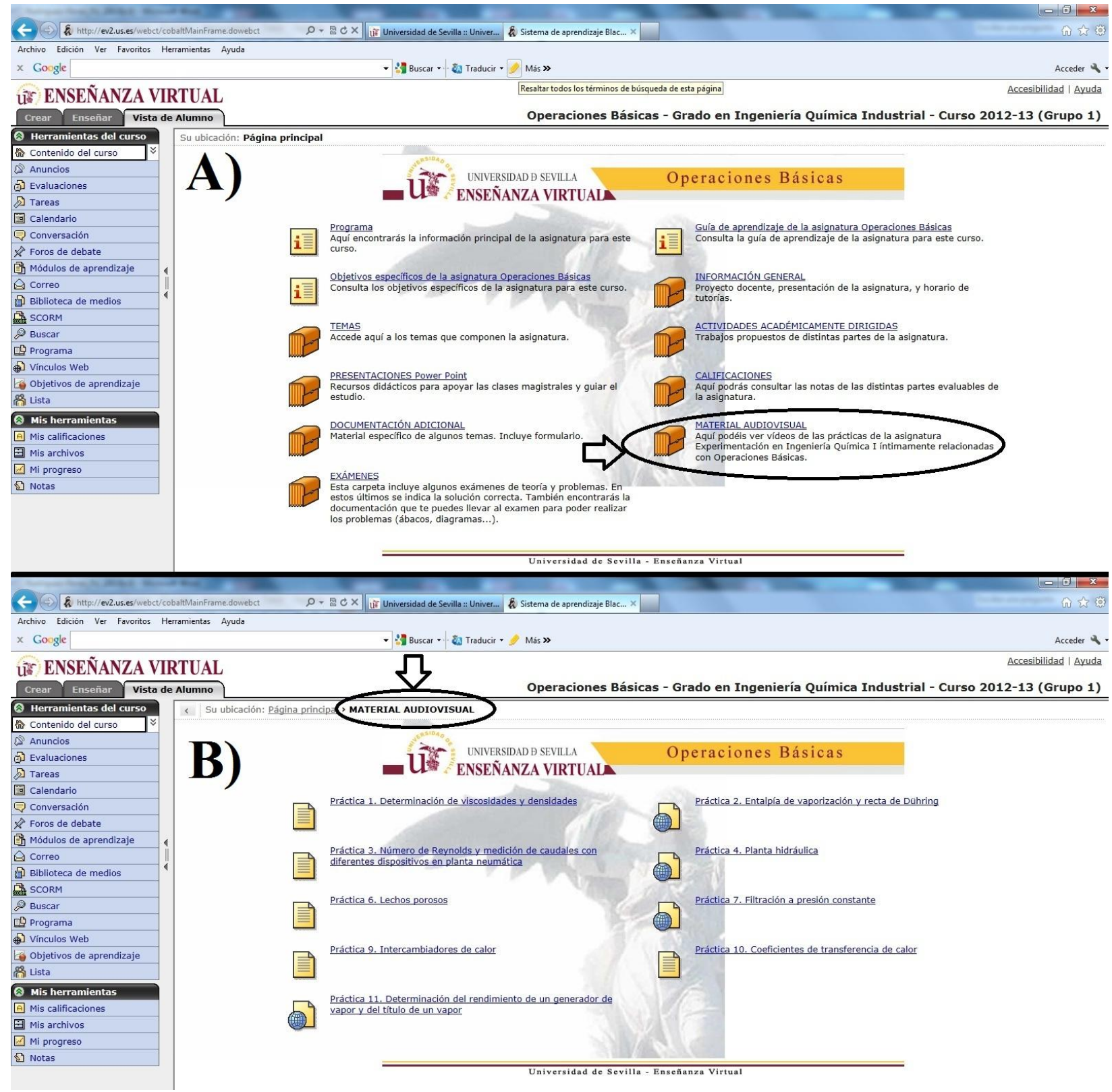

Fig. 1: Acceso al material audiovisual en WebCT. A) Página principal del Contenido del Curso; B) Contenido de la carpeta Material Audiovisual.

Se editan y suben a la plataforma de enseñanza virtual 9 videos (Figura 1), que engloban los fundamentos teóricos sobre: la determinación de viscosidades y densidades; la entalpía de vaporización y la recta de Dühring; la medición de caudales y el número de Reynolds; la mecánica de fluidos aplicada a una planta hidráulica a escala de laboratorio; la filtración a presión constante; los lechos porosos; los intercambiadores de calor; los coeficientes de transferencia de calor; y la determinación del rendimiento de un generador de vapor y del título de un vapor.

El guión de los vídeos es igual para todos ellos: se comienza con la definición teórica del proceso, seguido del fundamento matemático necesario para el cálculo de las propiedades que se estudian en los diferentes procesos (viscosidades, caudales, variaciones de presión, coeficientes individuales de transmisión de calor, etc.). Después de ello, se observan secuencias de vídeo realizando dichas mediciones, preparando los equipos necesarios y/o identificando los aparatos de medida, así como las plantas a escala de laboratorio utilizadas. 
Una vez que el alumno accede a la carpeta del material audiovisual complementario, tiene a su disposición 9 vídeos. Todos ellos se titulan de forma muy descriptiva sobre el proceso que desarrolla (por ejemplo, Lechos porosos). Son vídeos de corta duración (tiempo medio de 3 minutos y 40 segundos), en los que, en primer lugar se realiza una definición teórica, seguida del desarrollo práctico en laboratorio. Siguiendo con el ejemplo de la práctica de Lechos Porosos, en primer lugar, se define qué son los lechos porosos y la fórmula para poder calcular la porosidad de un lecho (Figura 2a); por último, se muestra cómo se toman los datos necesarios, en el laboratorio, para calcular las densidades aparente y real de las partículas que forman el lecho poroso (Figura $2 \mathrm{~b}$ y $2 \mathrm{c}$ ).

Los vídeos elaborados permiten la utilización de distintos recursos como imágenes, gráficos y textos que captan la atención del alumno. El objetivo del uso de dicho material audiovisual complementario es la comprensión de los principios teóricos a través de la visualización de su aplicación práctica. Se potencia el repaso de los conceptos de la clase de teoría mediante un estímulo visual que, junto con la atención del alumno, facilita la asimilación de la operación básica a estudiar. Es por ello que, con la aplicación de las metodologías TIC, en la que los alumnos ven y dan sentido práctico a lo estudiado en teoría, pueden reforzar su comprensión y asimilación a largo plazo.

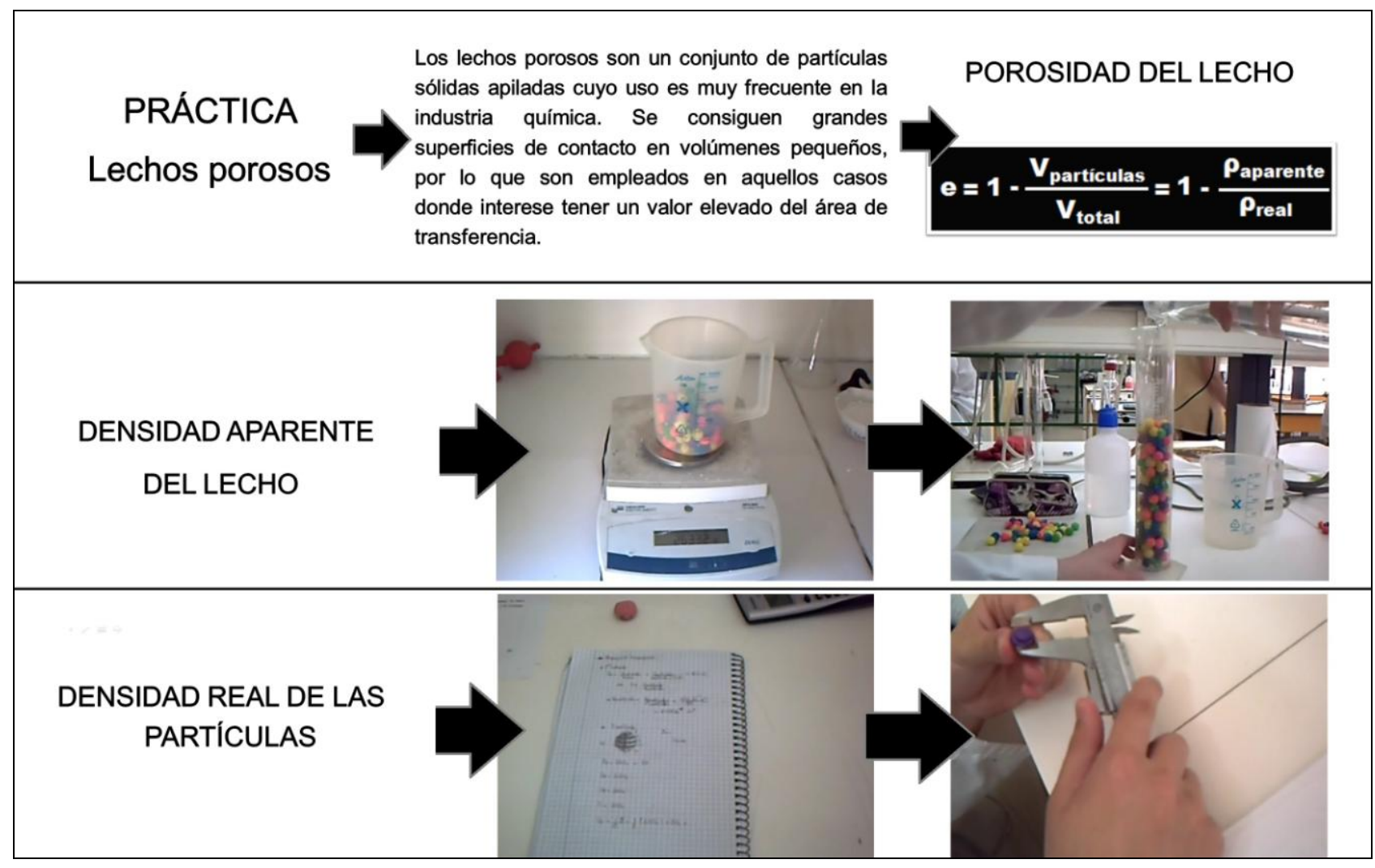

Fig. 2: Algunas secuencias de la práctica Lechos porosos.

Una de las ventajas añadidas del uso de las metodologías TIC a través de la Plataforma de Enseñanza Virtual de la Universidad de Sevilla es que permite cuantificar los accesos al material audiovisual complementario (Tabla 1). Esto se realiza mediante la herramienta del profesor denominada "Seguimiento: Informe de utilización de elementos del curso".

Tabla 1. Informe de utilización del elemento del curso MATERIAL AUDIOVISUAL.

\begin{tabular}{|c|c|c|}
\hline Visitas & Tiempo medio por visita & Tiempo total \\
\hline 17 & $00: 00: 08$ & $00: 02: 22$ \\
\hline
\end{tabular}

Dado que el tiempo medio por visita es de tan solo 8 segundos, mientras que el tiempo medio de los vídeos es de 3 minutos y 40 segundos, es obvio que los alumnos no han utilizado este material y el número de visitas se correspondería con las entradas del profesor en la pantalla "vista del alumno" para comprobar la correcta subida a red del material. 
El $100 \%$ de los alumnos encuestados opinaron que el material es interesante, sin embargo, ningún alumno accedió a los contenidos. En la Figura 3 se recogen los principales motivos expresados por los alumnos. Como puede observarse en la Figura 3, el 58.8\% de los alumnos indicaron que no tienen tiempo para ver un material complementario, a pesar de que a priori podría ser interesante. Un $11.8 \%$ puso de manifiesto que el resto de contenidos de la asignatura era suficiente, para afrontarla con éxito académico. Mientras que para un $5.9 \%$ era incluso excesivo. Otro $17.6 \%$ contestó que no había visto la carpeta y, por último, un $5.9 \%$ no contestó (NC).

En relación al rendimiento académico, los alumnos alcanzaron un nivel importante, con un $83 \%$ de aprobados sobre el total de presentados en el curso 2012-2013, siendo el 50\% de los aprobados con la calificación de notable (7 a 8,9) sobre 10. Si se comparan estos datos con los obtenidos en el curso 2010 2011 (en el curso 2011-2012 no se impartió esta asignatura por extinción del anterior Plan de Estudios 2001), $76 \%$ de aprobados sobre el total de presentados siendo el $47 \%$ con la calificación de notable, son ligeramente superiores. En este sentido, no hay correlación entre cantidad de alumnos que aprobaron el curso con la utilización del material audiovisual.

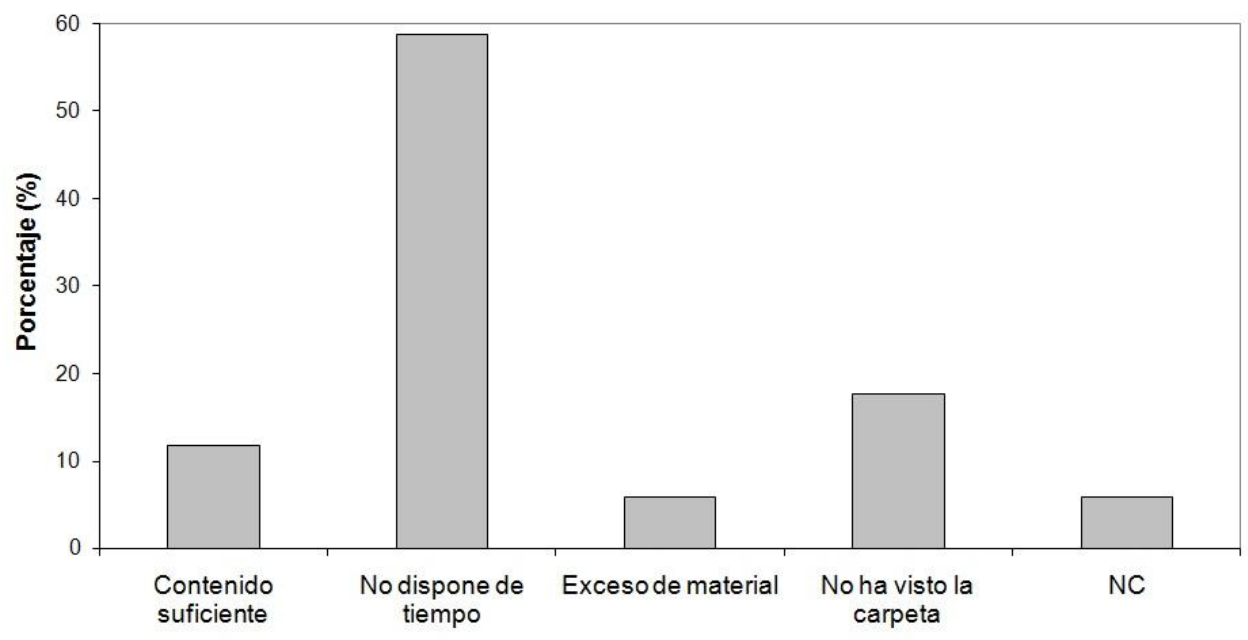

Fig. 3: Motivo dado por el alumno para explicar la no utilización del material audiovisual.

Es necesario señalar que, si bien la asignatura $\mathrm{OB}$ no tiene prerrequisitos académicos, se ha ido aumentado la exigencia progresivamente en los últimos años. Esto hace que gran parte de los estudiantes matriculados tengan ya asignaturas de cursos anteriores que se consideran claves para cursarla con éxito. En el curso académico 2012-2013 esta tendencia se ha visto incluso aumentada pues, debido al aumento del precio de las tasas académicas en segunda y siguientes convocatorias, los alumnos han preferido aprobar asignaturas de primer y segundo curso pendientes antes de matricularse por primera vez en asignaturas de tercero. Esto genera una población muy homogénea en cuanto a formación previa y un desarrollo del curso también muy homogéneo, ya que los estudiantes progresan adecuadamente y de manera similar.

Un hecho comparable ha sido también constatado por otros autores. Inzunza et al. (2012) señalan que en una población de estudiantes heterogénea (con diferencias de dominio de contenidos previos), contrariamente a lo esperado, los estudiantes con mejores calificaciones no son los usuarios más frecuentes de su curso virtual. Estos autores concluyen que aquellos alumnos con una mejor y mayor formación previa no requieren usar con frecuencia los recursos virtuales, siendo innecesarias herramientas complementarias para fortalecer su aprendizaje.

Otro aspecto a tener en cuenta es el alto grado de presencialidad del Grado en Ingeniería Química Industrial, con un promedio de 20 horas a la semana de clase (común a la mayoría de las ingenierías en España), mientras que en Estados Unidos el promedio de horas es de 16, en Israel es de 12 e incluso en otros países llegan a ser de 8 horas por semana (Marín, 2003).

Es necesario puntualizar que, durante varios años, la asignatura $\mathrm{OB}$ en WebCT ha sido ampliada, reformada y mejorada, por lo que la percepción del alumno es que se trata de una asignatura muy completa (Arnáiz y Ojeda, 2008, 2009, 2010). Elaborar y añadir más material, si bien puede ser interesante desde un punto de vista formal, en la práctica puede ser ineficaz por sobresaturación de contenidos o herramientas. No hay que olvidar que, durante el primer cuatrimestre de tercer curso, los alumnos cursan un mínimo de 4 asignaturas. El tiempo disponible se convierte, entonces, en un factor limitante a la hora de dedicar un 
esfuerzo extra a una asignatura. Algo similar es comentado por Muñoz-Cano et al. (2012). De esta manera, la estructura curricular actual de la titulación, con un alto número de horas presenciales, derivaría en la dificultad para el desarrollo de un proceso de innovación educativa con base en las TIC.

Desde el punto de vista del profesor, los resultados de este estudio tienen implicaciones importantes. Existe, por una parte, interés e incluso presión institucional y curricular por elaborar materiales en red: convocatorias de proyectos de innovación y mejora docente y méritos evaluables para optar a plazas de profesorado universitario. Sin embargo, y como se indicó con anterioridad, las TIC no son la panacea para solucionar cualquier problema educativo (Jung, 2005). Será tarea del profesor valorar en cada asignatura el tiempo, esfuerzos y recursos empleados en elaborar material TIC para un progreso óptimo tanto del alumno en su formación como del profesor en su carrera académica. El proceso de enseñanza-aprendizaje sigue siendo un reto y un desafío apasionantes.

\section{CONCLUSIONES}

Los alumnos valoran positivamente materiales audiovisuales en plataformas virtuales que potencien el proceso de enseñanza-aprendizaje.

Los alumnos no usan los nuevos recursos de aprendizaje disponibles en la asignatura virtual debido a una sobrecarga en las horas presenciales y no presenciales de trabajo autónomo durante el curso.

Los alumnos de nivel académico medio-alto no requieren recursos virtuales como complemento para fortalecer su aprendizaje.

La actual estructura curricular del Grado en Ingeniería Química Industrial, con una alta presencialidad, dificulta el empleo de herramientas nuevas por parte de los alumnos.

Para un mayor impacto de las TIC sería necesario un cambio orientado a una menor presencialidad del alumno.

El profesor debe valorar, en cada asignatura, el coste docente y económico empleados en elaborar material TIC. Y ello dentro del contexto de la totalidad de las asignaturas que constituyen la titulación y de las necesidades educativas del grupo de estudiantes en particular.

\section{AGRADECIMIENTOS}

Los autores agradecen el apoyo de la Universidad de Sevilla que financió este proyecto a través del I Plan Propio de Docencia (Convocatoria 2011- Proyectos de Innovación y Mejora Docente).

\section{REFERENCIAS}

Arnáiz, C y Ojeda, E. Elaboración de Materiales en Red: Asignatura Operaciones Básicas. Plan de Renovación de las Metodologías Docentes. Universidad de Sevilla. ISBN: 978-84-691-1864-1. Secretariado de Recursos Audiovisuales y Nuevas Tecnologías. (2008)

Arnáiz, C y Ojeda, E. Elaboración de Materiales en Red: Asignatura Operaciones Básicas. Open Course Ware. Universidad de Sevilla. http://ocwus.us.es/arquitectura-e-ingenieria/operacionesbasicas/Course_listing. (2009)

Arnáiz, C y Ojeda, E. Elaboración de Materiales en Red: Asignatura Operaciones Básicas. Plan de Renovación de las Metodologías Docentes. Universidad de Sevilla. ISBN: 978-84-693-8192-2. Secretariado de Recursos Audiovisuales y Nuevas Tecnologías. (2010)

Babich, A., Mavrommatis, K.T.H. Teaching of complex technological processes using simulations. Int. J. Eng. Educ. 25 (2), 209-220. (2009)

Barak, M. Transition from traditional to ICT-enhanced learning environments in undergraduate chemistry courses. Computers \& Education 48, 30-43. (2007)

Domingues, L., Rocha, I., Dourado, F., Alves, M. and Ferreira, E. C. Virtual laboratories in (bio) chemical engineering education. Education for Chemical Engineers, 5, 22-27. (2010) 
EPS. ¿En qué consisten los estudios de Grado de la Rama Industrial? http://www.eps.us.es/docencia/titulaciones/recursos/grados/rama-industrial.pdf. Acceso: 28 de mayo (2013).

García, I. y Gracia, J. A Methodology Based on Effective Practices to Develop Educational Software. Computación y Sistemas, 11 (4), 313-322. (2008)

Inzunza, B.C., (1); Rocha, R.A., Márquez, C.G. y Duk, M.S. Asignatura Virtual como Herramienta de Apoyo en la Enseñanza Universitaria. Formación Universitaria, 5 (4), 3-14. (2012)

Jung, I. ICT-Pedagogy Integration in Teacher Training: Application Cases Worldwide. Educational Technology \& Society, 8 (2), 94-101. (2005)

Jyothi, S., McAvinia, C. y Keating, J. A visualization tool to aid exploration of students' interactions in asynchronous online communication. Computers and Education, 58 (1), 30-42. (2012)

Komulainen, T.M., Enemark-Rasmussenb, R., Sin, G., Fletcherc, J.P., Cameron, D. Experiences on dynamic simulation software in chemical engineering education. Education for Chemical Engineers, 7, 153-162. (2012)

León-Mora, C., Camarillo-Casado, J., Ramos-Gómez, M., Sánchez-Aguilar, M.A. La enseñanza virtual en la Universidad de Sevilla. Pixel-Bit. Revista de Medios y Educación, 32, 7-20. (2008)

Maceiras, R., Cancela, A. y Goyanes, V. Aplicación de Nuevas Tecnologías en la Docencia Universitaria. Formación Universitaria, 3 (1), 21-26. (2010)

Marín Uribe, R. (2003). La docencia centrada en el aprendizaje. En: El modelo educativo de la UACH. Elementos para su construcción. Universidad Autónoma de Chihuahua, México. http://www.uach.mx/academica_y_escolar/modelo_educativo/2009/08/03/modeloeducativo-uach.pdf.

Acceso: 28 de mayo (2013).

Martín-Blas, T. y Serrano-Fernández, A. The role of new technologies in the learning process: Moodle as a teaching tool in Physics. Computers and Education, 52 (1), 35-44. (2009)

Muñoz-Cano, J.M., Córdova, J.A. y Priego, H. Dificultades y facilidades para el desarrollo de un proceso de innovación educativa con base en las Tecnologías de la Información y Comunicación (TIC). Formación Universitaria, 5(1), 3-12. (2012)

Sánchez, L. La Universidad de Sevilla fortalece su propuesta de Enseñanza Virtual con RODAS. http://servicio.us.es/gabcom/07-2011/28-julio.pdf. Acceso 28 de mayo (2013)

Solsona, N., Izquierdo, M., \& De Jong, O. Exploring the development of students conceptual profiles of chemical change. International Journal of Science Education, 25(1), 3-12. (2003)

US. Estudios y Acceso. http://www.us.es/estudios/index.html. Acceso: 28 de mayo (2013) 